\title{
Green shoots and double dips in the euro area. A real time
}

\section{measure*}

\author{
Maximo Camacho \\ Universidad de Murcia \\ mcamacho@um.es
}

Gabriel Perez-Quiros ${ }^{\dagger}$

Banco de España and CEPR

gabriel.perez@bde.es

Pilar Poncela

Universidad Autónoma de Madrid

pilar.poncela@uam.es

\begin{abstract}
To perform real-time business cycle inferences and forecasts of GDP growth rates in the euro area, we use an extension of the Markov-switching dynamic factor models that accounts for the specificities of the day to day monitoring of economic developments such as ragged edges, mixed frequencies and data revisions. We provide examples that show the nonlinear nature of the relations between data revisions, point forecasts and forecast uncertainty. According to our empirical results, we think that the realtime probabilities of recession inferred from the model are an appropriate statistic to capture what the press call green shoots and to monitor the double-dip recessions.
\end{abstract}

Keywords: Business Cycles, Output Growth, Time Series.

JEL Classification: E32, C22, E27

\footnotetext{
${ }^{*}$ We thank the comments from participants at 8th IIF Workshop (Paris) and from the editor and two anonymous referees. Maximo Camacho and Pilar Poncela thank MICINN for fiancial support: contract grants ECO2010-19830 and ECO2009-10287 and Banco Santander-CEAL respectively. Remaining errors are our own responsibility. The views in this paper are those of the authors and do not represent the views of Bank of Spain or the EuroSystem.

${ }^{\dagger}$ Corresponding Author: Gabriel Perez Quiros, Banco de España, Research Division, Monetary and Financial Studies Department, DG Economics, Statistics, and Research. Alcalá 48, 28014 Madrid, Spain. E-mail: gabriel.perez@bde.es
} 


\section{Introduction}

In 2008/09 the euro area faced the most serious economic recession since its creation. To stimulate the economic situation, a comprehensive set of aggressive fiscal stimulus measures and accommodative monetary policies were implemented either at the European level or by the State Members within the framework set up of the European Action Plan and the subsequent Commission Communications and ECB (European Central Bank) guidelines. These emergence measures led to a subsequent recovery period whose early signals were monitored by analysts, policy makers, and journalists with unprecedented interest. In those days, the term green shoots was largely popularized by them in reference to the signs of the economic recovery. However, the counter-cyclical measures adopted in the course of the recession gave way to a progressive deterioration of public finances in the euro area which, along with a wave of downgrading the government debt of some Member States, created an increasing loss of confidence among investors. As a result, the combination of tight credit conditions and fiscal austerity measures occurred in 2010 and 2011 put significant downward pressures on euro area GDP growth. At the end of 2011, the media started to talk about the possibility that the euro area region had probably entered a double-dip recession.

Needless is to say that the terms green shoots and double-dip recessions have not always been used with scientific criteria, mainly due to two reasons. First, the terms are very imprecise so they leave the users of the terms to identify where, when and how the recovery comes or ends depending basically on their own beliefs. Since the signals of recoveries do not appear in all the economic indicators with the same intensity at the same time in different sectors, the skeptical users will be inclined to accentuate the negative signals of some indicators while the optimistic users will be tempted to stress the positive signals of some others. Perhaps, it is the impreciseness of the definitions what also diminishes the meaning of international comparisons among these green shoots and double-dip recessions. Second, in the search of green shoots and double-dip recessions, the recent advances in information technologies make the number of indicators with relevant information about the economic evolution exponentially growing and with an unprecedented updating frequency. 
The cost of checking the publication calendar of these indicators, their latest releases and their subsequent revisions in real time, makes it very difficult the task of the analysts that try to check if the shoots are actually green or if a recession is coming back.

The purpose of this paper is to provide economic agents with statistical definitions of the terms green shoots and double-dip recessions which are very easy to interpret and can be timely and automatically updated. In particular, we define the term green shoot as a low probability of being in a recession at time $t$, which is inferred from the information available up to this period from a set of key economic indicators after a period of recession. In the same way, we consider that a region enters a double-dip recession when there are high recession probabilities which are preceded by a short period of low recession probabilities observed after a recession. These definitions overcome the two problems previously stated associated with the increasing use of the terms green shoots and double-dip recessions. First, the probability of recession is a precise term. The inferences about the state of the cycle are computed from a statistical model applied to data which are then transparent and objective. In addition, since recession probabilities are free of units of measurement, international comparisons are easily allowed. Second, since the inference is performed from a set of economic indicators that are considered as representative of the overall economic activity, the resulting recession probabilities become "sufficient statistics" for the analysts who benefit from the subsequent saving time and cost reduction in monitoring the euro area business cycles.

To compute recession probabilities in the euro area timely, we use an extension of the Markov-switching dynamic factor model. Initially, these models were developed by Kim and Yoo (1995), Chauvet (1998) and Kim and Nelson (1998) to combine the dynamicfactor approach, which captures the notion of comovements across economic indicators, and the Markov-switching framework, which captures the regime shifts observed in the dynamics of these indicators. Camacho, Perez-Quiros and Poncela (2012a) found that this fully nonlinear multivariate specification outperforms the "shortcut" of using a linear factor model to obtain a coincident indicator which is then used to compute the Markovswitching probabilities from univariate nonlinear models. Chauvet and Hamilton (2006), Chauvet and Piger (2008), and Hamilton (2011) examined the empirical reliability of these 
models in computing real-time inferences of the US business cycle states. Camacho, PerezQuiros and Poncela (2012b) extended the Markov-switching dynamic factor model to deal with the typical difficulties of the timely day to day monitoring of the economic activity such as mixing frequencies and ragged ends.

Following these lines, we extend the linear Euro-STING dynamic factor model suggested by Camacho and Perez-Quiros (2010) to deal with Markov-switching dynamics. Using the nonlinear extension advocated by Camacho, Perez-Quiros and Poncela (2012b) of the approximate linear Kalman filter suggested by Mariano and Murasawa (2003), the model is able to handle quarterly and monthly indicators and to fill in the gaps that characterize the asynchronous data publication. Notably, we show in this paper strong evidence in favor of the nonlinear nature of the data generating process.

The model is applied to euro area data to obtain an indicator of the overall economic activity and to compute business cycle inferences. Our findings point out that the model exhibits a remarkably ability to track the CEPR (Center for Economic Policy Research) Business Cycle Dating Committee chronology as captured in the state probabilities for the overall economic indicator. Unlike the CEPR committee, our method dates in terms of months rather than quarters. However, in dating the euro area business cycles, the paper should not be read as a substitutive but rather a complementary tool to other dating methods such as those revised in Anas, Billio, Ferrara and Lo Duca (2006).

In spite of its outstanding performance in dating the historical euro-area business cycles, the usefulness of the model is the significant improvements over the Dating Committee in the speed with which business cycle peaks and troughs are identified. To examine the timeliness of the model to automatically provide early warning signals about the economic downturns in the euro area, we construct a real-time dataset whose vintages were collected for this article and have not been applied in any other analysis of real-time business cycle dating. The dataset is used to develop several forecasting exercises which lead to the following interesting results. First, we show that the model provides a significant improvement in the speed with which the euro area business cycle turning points are identified. Although the Committee's mission is to establish the chronology of the euro area business cycle, it is likely more concerned with establishing the correct turning point dates than es- 
tablishing these dates quickly and, therefore, peak and trough dates are often determined with a substantial lag. ${ }^{1}$ Notably, our model is able to identify the euro area turning points almost in real time while exhibiting a low rate of false signals.

In addition, the Committee members have the possibility of partly basing their decisions on judgements. Consequently, the Committee's decisions represent the consensus of individuals and cannot be easily replicated. By contrast, our proposal seeks to avoid these problems by using a simple algorithm which, while keeping the necessary speed for the real-time monitoring in order to identify the turning points in the euro area, has the advantage of computing inferences from a specific model which can be evaluated in terms of transparency, replicability and decomposition of the informational content of all the macroeconomic data released.

Second, our article makes an important empirical contribution to the existing literature on dynamic factor models regarding the analysis of the relative ability of linear versus Markov-switching factor models on forecasting GDP growth. To our knowledge, this is the first attempt to investigate this topic since the previous literature was only concerned on evaluating the accuracy of these nonlinear models in computing business cycle inferences. Interestingly, we find that the nonlinear model performs similarly to the linear model in terms of computing short-term forecasts of the euro area GDP growth rates in real time, although the nonlinear specification has the advantage of computing timely inferences about the euro-area business cycles.

The structure of this paper is organized as follows. Section 2 outlines the model and discusses some econometric details regarding the extension of Markov-switching dynamic factor models to account for some particularities of real time forecasting. Section 3 evaluates the empirical reliability of the model in within sample and real-time exercises. Section 4 concludes.

\footnotetext{
${ }^{1}$ For instance, the Committee concluded on March, 31th 2009 that economic activity in the euro area peaked in the first quarter of 2008 and determined on October, 4th 2010 that a trough in economic activity occurred in the second quarter of 2009.
} 


\section{The model}

In this section, we briefly describe a small-scale dynamic factor model in which the business cycle indicators depend on a common factor, which evolves according to Markov-switching dynamics, and individual idiosyncratic components. ${ }^{2}$ The model is flexible enough to account for mixing frequencies, data revisions and unsynchronized data releases.

\subsection{Mixing frequencies}

The fact that some economic indicators are available monthly while others are available quarterly raises the question of how to combine them into a unified forecasting model. Quarterly series that refer to stocks can be converted easily in monthly observations since they simply refer to quantities that are measured at a particular time and do not require any time restriction. Accordingly, these series can be treated as observed the month that they are issued and as unobserved otherwise. However, flow variables are measured during some time periods and must be temporally aggregated. Mariano and Murasawa (2003) describe a time aggregation which is based on the notion that quarterly time series can be viewed as sums of underlying monthly series in the corresponding quarter. Assuming that arithmetic means can be approximated by geometric means, quarter-on-quarter growth rates $\left(g_{t}\right)$ of quarterly series are weighted averages of the month-on-month past growth rates $\left(x_{t}\right)$ of the (assumed to be known) monthly underlying series

$$
g_{t}=\frac{1}{3} x_{t}+\frac{2}{3} x_{t-1}+x_{t-2}+\frac{2}{3} x_{t-3}+\frac{1}{3} x_{t-4} .
$$

In this context, it is worth mentioning that although the influential proposal of Aruoba, Diebold and Scotti (2009a) early used polynomial detrending series to avoid approximations, they recently acknowledged (Aruoba, Diebold and Scotti, 2009b) that this leads to undesirable time series characteristics. Accordingly, their aggregation of flow variables used to continuously update their business conditions index maintained by the Federal Re-

\footnotetext{
${ }^{2}$ Interested readers on large-scale dynamic factor models are referred to the excellent survey by Stock and Watson (2011). In this context, it is worth pointing out that some recent papers (see Boivin and Ng, 2006, or Alvarez, Camacho and Perez Quiros, 2012) show that more data are not always better for factor analysis.
} 
serve Bank of Philadelphia is nowadays only approximate, as in Mariano and Murasawa (2003).

The weighted averaged expression implies that the model must handle missing values since the monthly series of quarterly growth rates, $g_{t}$, is only available once every three monthly outcomes. These missing data and the absence of the latest releases due to data publication delays can both be treated in a similar manner.

\subsection{Data revisions}

The fact that economic data are frequently revised complicates the day to day monitoring of the economic activity since revisions change the data input into forecasting models. In the euro area, Eurostat revises twice the GDP growth figures in its official data release process. ${ }^{3}$ The flash estimate, $y_{t}^{f}$, appears about 45 days after the end of the respective quarter. Since it is based on preliminary information, Eurostat publishes the first estimate about 20 days after which relies on more complete data. Finally, the second estimate of GDP growth rate, $y_{t}^{2 n d}$, incorporates an additional revision about 40 days after the first. According to this revision process, let us call $e_{1}$ the revision between the flash and the first, and $e_{2}$ the revision between the first and the second.

In this paper, we follow Evans (2005) and Coenen, Levin, Wieland (2005) to consider that preliminary advances are noisy signals of revised data:

$$
\begin{aligned}
y_{t}^{f} & =y_{t}^{2 n d}+e_{1 t}+e_{2 t}, \\
y_{t}^{1 s t} & =y_{t}^{2 n d}+e_{2 t},
\end{aligned}
$$

where $e_{1 t}$ and $e_{2 t}$ are independent zero mean revision shocks with variances $\sigma_{e_{1}}^{2}$ and $\sigma_{e_{2}}^{2}$, respectively. ${ }^{4}$ Camacho and Perez-Quiros (2010) show empirical evidence to be confident that this specification is a reasonable representation of the data revision process.

It is important to remark that, starting with the publication of the flash estimate for the fourth quarter of 2010 on February 15th 2011, Eurostat is implementing a new release

\footnotetext{
${ }^{3}$ Other major revisions can also be modeled. However, in this paper we only consider the official GDP release calendar.

${ }^{4}$ For simplicity, we assume that $e_{1 t}$ and $e_{2 t}$ are independent.
} 
policy for quarterly national accounts. From this day, there are two new releases each quarter, instead of three. The preliminary release, called the flash estimate, is published about 45 days after the end of the respective quarter. This is followed by the second estimate, which is published with a delay of 65 days. In practice, the change implies substituting the first by the second. According to this change, the model was modified since February, 15th 2011 in the real-time forecasting analysis.

\subsection{Ragged edges}

In spite of the technical difficulties associated to the real time assessments of the economic activity that have been discussed below, real-time forecasters have to deal with the typical lack of synchronicity in data publication. Following a typical release calendar, monthly indicators are published much more timely than quarterly series. In addition, although indicators based on surveys (soft indicators) are more promptly issued than economic activity indicators (hard indicators), their samples are usually shorter.

This implies that standard Markov-switching dynamic factor models are of limited use in real-time analyses since they were originally designed to deal with balanced panels of business cycle indicators. Not accounting for this publication pattern would imply that real-time forecasters who develop early assessments of economic developments from balanced panels of data will unavoidably incur in one of the two following substantial costs. The first appears when the forecasts are made from the latest available balanced panel. In this case, the forecasts lose the latest and most valuable information contained in the promptly issued indicators at the time of the assessments. The second is that of being late when the analysts decide to wait until all the business cycle indicators become available and the inferences are then actually referred to the past.

Camacho, Perez-Quiros and Poncela (2012b) proposed to fill in the missing observations that appear from mixing frequencies and ragged ends with random numbers that are extracted from a random variable whose distribution is independent of the model parameters and rewriting the measurement equation appropriately to get a time-varying Kalman filter that skips the random numbers. They show that the parameters that maximize the likelihood and the inferences about the business cycle states are not affected by these 
filled in data. This leads the forecasting procedure to become an extremely easy exercise. Computing $k$-period ahead forecasts reduces to add $k$ rows of missing data at the end of the dataset which will automatically be replaced by forecasts inside the model.

\subsection{Specification of the model}

The Markov-switching dynamic factor model consists of a factor model which decomposes the dynamics of the business cycle indicators into two components. The first component is a common factor which captures the occasional discrete variations in the dynamic features of the business cycle indicators. The second component refers to the idiosyncratic dynamics of each indicator and is modelled by using the standard techniques of linear autoregressive time series.

To be specific, in this model the common factor, $f_{t}$, is driven by an unobservable state variable $s_{t}$ :

$$
f_{t}=\alpha_{s_{t}}+a_{1} f_{t-1}+\ldots+a_{m_{1}} f_{t-m_{1}}+\epsilon_{t}^{f},
$$

where $\epsilon_{t}^{f} \sim$ i.i.d.N $\left(0, \sigma_{f}^{2}\right)$, and $m_{1}$ is the autoregressive order. In this paper, $s_{t}$ is assumed to evolve according to an irreducible 2-state Markov chain whose transition probabilities are defined by

$$
p\left(s_{t}=j \mid s_{t-1}=i, s_{t-2}=h, \ldots, \chi_{t-1}\right)=p\left(s_{t}=j \mid s_{t-1}=i\right)=p_{i j},
$$

where $i, j=0,1$, and $\chi_{t}$ is referred to the information set up to period $t$.

In the related literature, several specifications of the nonlinear dynamics of the common factor have been suggested. Kim and Yoo (1995) and Chauvet (1998) allowed the intercept term to be regime dependent. In the specification of Kim and Nelson (1998) it is the mean instead of the intercept what is allowed to exhibit regime shifts. In this paper, we follow Camacho and Perez-Quiros (2007) and assume that the factor dynamics can be captured by shifts between the business cycle states, so we set the autoregressive coefficients to zero. Within this framework, we can label $s_{t}=0$ and $s_{t}=1$ as the expansion and recession states at time $t$ if $\alpha_{0}>0$ and $\alpha_{1}<0$. Hence, the common factor is expected to exhibit positive growth rates in expansions and lower (usually negative) growth rates in recessions. 
To specify the dynamic factor model of our data set (that it will include variables such as flash, first, second, employment, and hard and soft indicators), let us first assume that missing data do not appear in the dataset so that quarterly series are observed monthly and the corresponding vintage panels are balanced. We also assume that the factor captures the common dynamics in the growth rates of real activity data. However, since survey indicators in Europe are designed to capture annual growth rates of the reference series (see European Commission, 2006), we impose that the levels of soft indicators depend on the sum of current values of the common factor and their latest eleven lagged values.

Let us collect the $r_{h}$ hard indicators in the vector $Z_{t}^{h}$ and the $r_{s}$ soft indicators in the vector $Z_{t}^{s}$. Let $l_{t}$ be the quarterly employment growth rate, and let $u_{1 t}, u_{2 t}, U_{t}^{h}$, and $U_{t}^{s}$ be the scalars and $r_{h}$-dimensional and $r_{s}$-dimensional vectors which determine the idiosyncratic dynamics in the model. The dynamics of the business cycle indicators can be stated as

$$
\left(\begin{array}{c}
y_{t}^{2 n d} \\
Z_{t}^{h} \\
Z_{t}^{s} \\
l_{t} \\
y_{t}^{1 s t} \\
y_{t}^{f}
\end{array}\right)=\left(\begin{array}{c}
\beta_{1}\left(\frac{1}{3} f_{t}+\frac{2}{3} f_{t-1}+f_{t-2}+\frac{2}{3} f_{t-3}+\frac{1}{3} f_{t-4}\right) \\
\beta_{2} f_{t} \\
\beta_{3} \sum_{j=0}^{11} f_{t-j} \\
\beta_{4}\left(\frac{1}{3} f_{t}+\frac{2}{3} f_{t-1}+f_{t-2}+\frac{2}{3} f_{t-3}+\frac{1}{3} f_{t-4}\right) \\
\beta_{1}\left(\frac{1}{3} f_{t}+\frac{2}{3} f_{t-1}+f_{t-2}+\frac{2}{3} f_{t-3}+\frac{1}{3} f_{t-4}\right) \\
\beta_{1}\left(\frac{1}{3} f_{t}+\frac{2}{3} f_{t-1}+f_{t-2}+\frac{2}{3} f_{t-3}+\frac{1}{3} f_{t-4}\right)
\end{array}\right)+\left(\begin{array}{c}
\frac{1}{3} u_{1 t}+\frac{2}{3} u_{1 t-1}+u_{1 t-2}+\frac{2}{3} u_{1 t-3}+\frac{1}{3} u_{1 t-4} \\
U_{t}^{h} \\
U_{t}^{s} \\
\frac{1}{3} u_{2 t}+\frac{2}{3} u_{2 t-1}+u_{2 t-2}+\frac{2}{3} u_{2 t-3}+\frac{1}{3} u_{2 t-4} \\
\frac{1}{3} u_{1 t}+\frac{2}{3} u_{1 t-1}+u_{1 t-2}+\frac{2}{3} u_{1 t-3}+\frac{1}{3} u_{1 t-4} \\
\frac{1}{3} u_{1 t}+\frac{2}{3} u_{1 t-1}+u_{1 t-2}+\frac{2}{3} u_{1 t-3}+\frac{1}{3} u_{1 t-4}
\end{array}\right)+\left(\begin{array}{c}
0 \\
0 \\
0 \\
0 \\
0 \\
e_{2 t} \\
e_{1 t}+e_{2 t}
\end{array}\right),
$$

where $U_{t}^{h}=\left(v_{1 t}, \ldots, v_{r_{h}}\right)^{\prime}, U_{t}^{s}=\left(v_{r_{h}+1 t}, \ldots, v_{r t}\right)^{\prime}$, and $r=r_{h}+r_{s}$. The factor loadings, $\beta=\left(\begin{array}{llll}\beta_{1} & \beta_{2}^{\prime} & \beta_{3}^{\prime} & \beta_{4}\end{array}\right)^{\prime}$, measure the sensitivity of each series to movements in the latent factor and have dimensions that lead them to be conformable with each equation. 
The complete dynamics of the model is achieved by assuming that

$$
\begin{aligned}
& u_{1 t}=b_{1} u_{1 t-1}+\ldots+b_{m_{2}} u_{1 t-m_{2}}+\epsilon_{t}^{u_{1}}, \\
& v_{j t}=c_{j 1} v_{j t-1}+\ldots+c_{j m_{3}} v_{j t-m_{3}}+\epsilon_{t}^{v_{j}}, \\
& u_{2 t}=d_{1} u_{2 t-1}+\ldots+d_{m_{4}} u_{2 t-m_{4}}+\epsilon_{t}^{u_{2}},
\end{aligned}
$$

where $\epsilon_{t}^{u_{1}} \sim$ i.i.d.N $\left(0, \sigma_{u_{1}}^{2}\right), \epsilon_{t}^{u_{2}} \sim$ i.i.d.N $\left(0, \sigma_{u_{2}}^{2}\right)$, and $\epsilon_{t}^{v_{j}} \sim$ i.i.d.N $\left(0, \sigma_{v_{j}}^{2}\right)$, with $j=$ $1, \ldots, r$. All the covariances are assumed to be zero and we set the variance of the noise associated to the common factor, $\sigma_{f}^{2}$, equal to one. ${ }^{5}$

Consider the following state space representation of the Markov-switching dynamic factor model

$$
\begin{aligned}
& Y_{t}=H_{t} h_{t}+w_{t}, \\
& h_{t}=\Lambda_{s_{t}}+F h_{t-1}+\xi_{t},
\end{aligned}
$$

where the state vector $h_{t}$ is $n \times 1$ and contains $f_{t}$ and its first 11 lags as well as the idiosyncratic components and their lags $\Lambda_{s_{t}}=\left(\begin{array}{cc}\alpha_{s_{t}} & 0_{1, n-1}\end{array}\right)^{\prime}, s_{t}=i, j$, and

$$
\left(\begin{array}{c}
w_{t} \\
\xi_{t}
\end{array}\right) \sim \operatorname{iidN}\left(0,\left(\begin{array}{cc}
R_{t} & 0 \\
0 & Q
\end{array}\right)\right) .
$$

The Appendix provides more details on the model structure and the specific forms of these matrices.

Let us now describe how to handle missing data. For this purpose, we substitute missing observations with random draws $\theta_{t}$ from $N\left(0, \sigma_{\theta}^{2}\right)$. This implies replacing the $i$-th row of $Y_{t}, H_{t}, w_{t}$ (denoted by $Y_{i t}, H_{i t}$ and $w_{i t}$ ) and the $i$-th element of the main diagonal of $R_{t}\left(R_{i i t}\right)$, by $Y_{i t}^{*}, H_{i t}^{*}, w_{i t}^{*}$, and $R_{i i t}^{*}$, respectively. The starred expressions are $Y_{i t}, H_{i t}, 0$, and 0 if the variable $Y_{i t}$ is observable at time $t$, and $\theta_{t}, 0_{1, n}, \theta_{t}$, and $\sigma_{\theta}^{2}$ in case of missing data. Accordingly, this transformation converts the model in a time-varying state space model with no missing observations and the nonlinear version of the Kalman filter can be directly applied to $Y_{t}^{*}, H_{t}^{*}, w_{t}^{*}$, and $R_{t}^{*}{ }^{6}$

\footnotetext{
${ }^{5}$ This identifying assumption is standard in dynamic factor models estimated in state space form using the Kalman filter.

${ }^{6}$ Alternatively, the problem of missing data could be treated by imposing infinite variances when the data is missing.
} 
To describe how the model can be estimated, let $h_{t \mid \tau}^{(i, j)}$ be the forecast of $h_{\tau}$ based on information up to period $\tau$ and the realized states $s_{t-1}=i$ and $s_{t}=j$, and let $P_{t \mid \tau}^{(i, j)}$ be its covariance matrix. The prediction equations become

$$
\begin{aligned}
h_{t \mid t-1}^{(i, j)} & =\Lambda_{j}+F h_{t-1 \mid t-1}^{i}, \\
P_{t \mid t-1}^{(i, j)} & =F P_{t-1 \mid t-1}^{i} F^{\prime}+Q,
\end{aligned}
$$

where $h_{t-1 \mid t-1}^{i}$ is the estimation of $h_{t}$ at time $t-1$ with information up to time $t-1$ if $s_{t-1}=i$ and $P_{t-1 \mid t-1}^{i}$ its mean squared error matrix defined in (18) and (19), respectively. The conditional one-step-ahead forecast errors are $\eta_{t \mid t-1}^{(i, j)}=Y_{t}^{*}-H_{t}^{*} h_{t \mid t-1}^{(i, j)}$ and $\zeta_{t \mid t-1}^{(i, j)}=$ $H_{t}^{*} P_{t \mid t-1}^{(i, j)} H_{t}^{* \prime}+R_{t}^{*}$ is its conditional variance. Hence, the log likelihood given $s_{t-1}=i$ and $s_{t}=j$ can be computed at each $t$ as

$$
l_{t}^{(i, j)}=-\frac{1}{2} \ln \left(2 \pi\left|\zeta_{t \mid t-1}^{(i, j)}\right|\right)-\frac{1}{2} \eta_{t \mid t-1}^{(i, j)^{\prime}}\left(\zeta_{t \mid t-1}^{(i, j)}\right)^{-1} \eta_{t \mid t-1}^{(i, j)}
$$

The updating equations become

$$
\begin{aligned}
h_{t \mid t}^{(i, j)} & =h_{t \mid t-1}^{(i, j)}+K_{t}^{(i, j)} \eta_{t \mid t-1}^{(i, j)}, \\
P_{t \mid t}^{(i, j)} & =P_{t \mid t-1}^{(i, j)}-K_{t}^{(i, j)} H_{t}^{*} P_{t \mid t-1}^{(i, j)},
\end{aligned}
$$

where the Kalman gain, $K_{t}^{(i, j)}$, is defined as $K_{t}^{(i, j)}=P_{t \mid t-1}^{(i, j)} H_{t}^{* \prime}\left(\zeta_{t \mid t-1}^{(i, j)}\right)^{-1}$.

It is worth pointing out that combining monthly and quarterly frequencies in this nonlinear framework typically leads to the curse of dimensionality problem. For instance, while the likelihood of a two-state DFM that only accounts for monthly indicators can be approximated by running two parallel Kalman filters in the simplest case, adding a quarterly indicator implies evaluating more than two parallel Kalman filters in the same simplest case. Since the factor can be in either of the two states, expansion or recession, and the monthly series of quarterly growth is a linear combination of the factor four lags, the process needs to account for up to $2^{5}=32$ different business cycle paths.

One immediate implication of this fact is that the empirical applications of this model will suffer from the curse of dimensionality problem. To overcome this problem, Camacho, Perez-Quiros and Poncela (2012b) design the following Monte Carlo exercise. Skipping the details, they generate 1000 sets of 3 monthly and 1 quarterly indicators of length 
$T=200$ which are restricted to follow a Markov-switching dynamic factor model. In these simulations, the generated business cycle dummies are assumed to follow the dynamic properties implicitly embedded in the NBER chronology. The generated datasets are then estimated and used to compute recession probabilities which are compared with the generated business cycle dummies. Using this framework, they show that approximating the time-consuming model that uses the 32 states actually required by a fast simplifying version that uses only the 2 initial states does not lead to large performance declines. According to their results, we use the simplification of estimating the 2-state simplification of the 32 states Markov-switching dynamic factor model. ${ }^{7}$

In addition, maximizing the exact log likelihood function of the associated nonlinear Kalman filter is computational bourdersome since at each iteration, the filter produces a 2 -fold increase in the number of cases to consider. The solution adopted in this paper is based on collapsing some terms of the former filter as proposed by Kim (1994) and used by Kim and Yoo (1995) and Chauvet (1998). In particular, the proposal of Kim (1994) is based on collapsing the posteriors $h_{t \mid t}^{(i, j)}$ and $P_{t \mid t}^{(i, j)}$ at the end of each iteration by using their weighted averages for $s_{t}=j$ where the weights are given by the probabilities of the Markov state:

$$
\begin{aligned}
h_{t \mid t}^{j} & =\frac{\sum_{s_{t-1}=0}^{1} p\left(s_{t}=j, s_{t-1}=i \mid \chi_{t}\right) h_{t \mid t}^{(i, j)}}{p\left(s_{t}=j \mid \chi_{t}\right)} \\
P_{t \mid t}^{j} & =\frac{\sum_{s_{t-1}=0}^{1} p\left(s_{t}=j, s_{t-1}=i \mid \chi_{t}\right)\left(P_{t \mid t}^{(i, j)}+\left(h_{t \mid t}^{j}-h_{t \mid t}^{(i, j)}\right)\left(h_{t \mid t}^{j}-h_{t \mid t}^{(i, j)}\right)^{\prime}\right)}{p\left(s_{t}=j \mid \chi_{t}\right)} .
\end{aligned}
$$

To conclude this section, let us point out one additional advantage of this proposal against standard Markov-switching dynamic specifications applied to balanced datasets: our model can easily compute GDP growth forecasts. Recall that our method mixes frequencies and fills in outliers following the rule of replacing missing by random numbers which allows us to include GDP growth as an additional business cycle indicator. In this

\footnotetext{
${ }^{7}$ To be sure that these results hold in our framework, we also performed the in-sample estimation of the 32-state model. The resulting recession probabilities were similar to those of the 2 -state simplification (correlation of 0.9 ).
} 
context, if we call $T$ the last month for which we have observed GDP growth, we call $h_{T+1 \mid T}^{(j)}$ the collapsed version of $h_{T+1 \mid T}^{(i, j)}$, and we call $h_{T+1 \mid T}^{(j)}(k)$ the $k$-th element of $h_{T+1 \mid T}^{(j)}$, taking into account that $h_{t}$ contains $f_{t}$ and its first 11 lags, as well as the idiosyncratic components and their lags, the forecasts for month $T+1$ when $s_{T+1}=j$ can be computed by the model as

$$
\begin{gathered}
y_{T+1 / T}^{2 n d, j}=\beta_{1}\left(\frac{1}{3} h_{T+1 \mid T}^{(j)}(1)+\frac{2}{3} h_{T+1 \mid T}^{(j)}(2)+h_{T+1 \mid T}^{(j)}(3)+\frac{2}{3} h_{T+1 \mid T}^{(j)}(4)+\frac{1}{3} h_{T+1 \mid T}^{(j)}(5)\right)+ \\
\left(\frac{1}{3} h_{T+1 \mid T}^{(j)}(13)+\frac{2}{3} h_{T+1 \mid T}^{(j)}(14)+h_{T+1 \mid T}^{(j)}(15)+\frac{2}{3} h_{T+1 \mid T}^{(j)}(16)+\frac{1}{3} h_{T+1 \mid T}^{(j)}(17)\right) .
\end{gathered}
$$

Using the matrix of transition probabilities, one can easily obtain $p\left(s_{T+1}=j, s_{T}=i \mid \chi_{t}\right)$ which can be used to compute $p\left(s_{T+1}=j \mid \chi_{t}\right)=\sum_{i} p\left(s_{T+1}=j, s_{T}=i \mid \chi_{t}\right)$ and the unconditional forecasts of GDP

$$
y_{T+1 / T}^{2 n d}=\sum_{j} p\left(s_{T+1}=j \mid \chi_{t}\right) y_{T+1 / T}^{2 n d, j}
$$

It is worth noting that these forecasts are easily computed in practice by including a missing observation $y_{T+1}^{2 n d}$ in the dataset since the model will automatically replace the missing by a dynamic forecast. Following the same reasoning, forecasts for longer horizons and forecasts for other indicators can be automatically computed.

\section{Empirical results}

\subsection{Data description}

The empirical analysis focuses on the thirteen business cycle indicators used in the linear Euro-STING dynamic factor model of Camacho and Perez-Quiros (2010). The authors found that the routinely updated short-term forecasts of euro area GDP produced by the linear model were very accurate and compare well with institutional forecasts such as (i) the European Commission's macroeconomic forecasts, (ii) the euro area GDP growth projection of the European Commission Directorate General for Economic and Financial Affairs (DG ECFIN), (iii) the economic forecasts that prepare jointly the German IFO institute, the French INSEE institute and the Italian ISAE institute (IFO-INSEE-INSAE), 
and (iv) the projections of the OECD Economic Outlook, who can combine the best forecasting tools with the possibility of incorporating their own judgement.

From a list of potential business cycle indicators, Camacho and Perez Quiros (2010) selected the indicators from successive enlargements of the single-index dynamic factor model proposed by Stock and Watson (1991) following three reasonable criteria. First, they must exhibit high statistical correlation with the euro area GDP growth rate. Second, they should be promptly available in the sample considered. Third, they must be relevant in the model from both theoretical and empirical points of view and must show explanatory power in terms of the estimated model. Therefore, the enlargements increased the proportion of the variance of GDP explained by the common factor while keeping their loading factors statistically significant.

The final set of business cycle indicators included in the model were (1) four quarterly series, second GDP growth releases, its two preliminary announcements flash and first, and employment, all of them in quarterly growth rates; (2) four monthly hard indicators, the euro area Industrial Production Index (IPI, excluding construction), the Industrial New Orders index (INO, total manufacturing working on orders), the euro area total retail sales volume, and extra-euro area Exports, all of them in monthly growth rates; and (3) five soft indicators, the euro-zone Economic Sentiment Indicator (ESI), the German business climate index (IFO), the Belgian overall business indicator (BNB), and the euro area Purchasing Managers confidence Indexes (PMI) in services and manufactures sectors, which are loaded in levels.

Our effective sample goes from April 1991 to November 2011. According to the standard analyses in the related literature, the data used in the empirical analysis are firstly standardized by substracting the sample mean from each variable and dividing by its standard deviation. Therefore, real-time forecasts are recursively computed by multiplying the initial forecasts of the model by the recursively updated standard deviations, and then adding the recursively updated means. 


\section{$3.2 \quad$ In-sample analysis}

The in-sample analysis was carried out with the vintage data set that was available on November, 24th 2011. The unsynchronized way on which data are published is illustrated in Table 1, which reports the latest available figures of each indicator. Since GDP and Employment releases appear quarterly, the two first months of each quarter are treated as missing data. Although it does not occur in this vintage, preliminary advances of GDP growth (flash and first) could be available before the publication of second GDP. Survey data have very short publishing delay of one (or even less) month while hard data are released with a relatively longer delay of about two months.

To provide evidence as to whether the model accords to the assumptions regarding regime switching, we perform several exercises. First, we assumed that the positive autocorrelation of the common factor can be captured by a recurrent sequence of shifts between two fixed equilibria of high and low means as an alternative to linear autoregressive processes. ${ }^{8}$ To examine this assumption, we estimated the common factors from both linear and Markov-switching dynamic factor models from the same dataset and we obtain that the sample correlation between both factors was $0.97 .^{9}$ This result is not surprising since Camacho and Perez-Quiros (2007) show that the persistence in a time series whose dynamics track the business cycle can be alternatively captured both by Markov-switching models and by linear autoregressive methods.

As a second exercise to assess the empirical reliability of the assumptions, we show that the factor actually exhibits business cycle dynamics. The maximum likelihood estimates of the parameters show that the factor is expected to be significantly positive (value of 0.24 with standard deviation of 0.09 ) in the state $s_{t}=0$ while it is significantly negative $(-2.57$ with standard deviation of 0.37$)$ in the state $s_{t}=1$. Accordingly, we can associate these states as expansions and recessions. In addition, each regime is highly persistent, with estimated probabilities of one regime to be followed by the same regime of 0.98 in the case of expansions and 0.91 in the case of recessions (standard deviations of 0.02 and 0.06 ,

\footnotetext{
${ }^{8}$ We imposed $m_{1}=0$ in (4).

${ }^{9}$ Factors extracted from linear and nonlinear filters lead to very similar graphs. They have been omitted to save space.
} 
respectively). Finally, another interesting business cycle implication of the Markov framework is that one can derive the expected number of quarters that the business cycle phases prevail. Conditional on being in state 0 , the expected duration of a typical expansion in the Euro area is $\left(1-\widehat{p}_{00}\right)^{-1}$ or about 50 months, and the expected duration of a recession is likewise $\left(1-\widehat{p}_{11}\right)^{-1}$ or 11.11 months. These estimates agree with the well-known fact that expansions are longer than contractions on average.

The model was also constructed under the assumption that the indicators share the underlying euro area aggregate economic activity dynamics whose pattern is captured by the common factor. Hence, although the scope of this paper is more ambitious than simply constructing a coincident index, we additionally check if the dynamics of the common factor are consistent with the euro area business cycles. For this purpose, the switching factor coincident index estimated in this paper is plotted in Figure 1 along with the Eurocoin, which is published each month by the CEPR and is considered the leading coincident indicator of the euro area business cycle. ${ }^{10}$ A visual inspection of the figure suggests that the common factor and the Eurocoin move together synchronously. Although the Eurocoin moves very smoothly since it is designed to track the medium term trend (by removing short-run fluctuations from a large dataset), the sample correlation between these two series is about 0.85 .

Remarkably, the figure also shows that there is a high commonality among their switching times. While both indicators fluctuate around their respective means, the broad changes of direction in these indicators seem to mark quite well the same cycles. In particular, they exhibited two periods of pronounced drops in dates for which GDP growth rates became worse significantly: 1992-1993 and 2008-2009. Interestingly, the deterioration in 2001 was milder and much shorter. Of special interest is the most recent period for which both indicators reached a peak in Summer 2011 and have declined since then.

To examine the dynamic correlation of the thirteen economic indicators and the factor, Table 2 reports the maximum likelihood estimates of the factor loadings (standard errors are in parentheses). The estimates are always positive and statistically significant, which agrees with the standard view that the indicators are procyclical. With respect to

\footnotetext{
${ }^{10}$ Using expression (1), the figure actually plots the "quarterly" transformation of the common factor.
} 
the size of the correlations, the economic indicators with larger factor loadings are those corresponding to IPI (0.49), INO (0.45), GDP (0.28) and Exports (0.27). Soft indicators exhibit much lower factor loadings, with a maximum of 0.09 in the case of PMI in manufactures. This result could erroneously be interpreted as a clear signal against the inclusion of the surveys as coincident indicators. In contrast, Camacho and Perez-Quiros (2010) show that the in-sample estimates of the factor loadings do not reflect the timely advantages of survey data that are observed in real-time exercises.

The Markov-switching dynamic factor specification developed in this paper provides a framework to date the historical euro area business cycle phases. For this purpose, we show in Figure 2 the monthly full sample smoothed inferences that the economy is in recession. To confront them with the data, this figure adds the quarterly GDP growth rates, which are estimated at monthly frequency from the model. ${ }^{11}$ The graph shows several shaded areas which indicate the recessions identified by the CEPR committee. For international comparisons, the US recessions dated by the NBER committee are also highlighted in the graph as dotted lines.

To start, the figure shows that the business cycle concordance between the euro area and the US has increased significantly in the Great Recession. The US clearly led the 1991 recession and the CEPR committee, in contrast to the NBER committee, acknowledged that there was not European recessions in 2001 or in 2003. However, the 2008-2009 recession become very synchronized since the US peak leads the European peak in only one quarter and their troughs occurs simultaneously in the second quarter of 2009.

Figure 2 shows that the recession probabilities inferred from our model create clear signals about the euro area business cycle states. High probabilities of recessions appear in 1992-1993 and 2008-2009 which correspond to low (or negative) growth. Although in these downturns the sharp transitions in recession probabilities from one regime to the other cohere strongly with the euro area business cycle turning points, our dating method seems to signal the 1993 trough earlier (about March) compared to the Committee's decision of dating the trough in the third quarter. Remarkably, our trough is in concordance with

\footnotetext{
${ }^{11}$ According to the model, GDP monthly estimates equal to the actual figures in the third month of each quarter.
} 
the evolution of the quarter-on-quarter GDP growth rates in euro area which fell $0.76 \%$ in the first quarter but rose $0.17 \%$ and $0.43 \%$ the second and third quarters, respectively. ${ }^{12}$

The main discrepancy between the US and euro-area recession dates occurs in the early 2000s. While the NBER declared a US recession in 2001, the CEPR acknowledged that the euro area experienced a prolonged pause in the growth of economic activity, rather than a full-fledged recession. In this period, our Markov-switching model produced probabilities of recession above 0.5 in September and October 2001 only, although the probabilities did not reach the levels of the 1992-1993 and 2008-2009 downturns. According to the low intensity and short duration of the increase in the recession probabilities, our model agrees with the CEPR's statement that the slower euro area growth rates observed in the early 2000s correspond, if any, with a very mild recession.

Interestingly, the recession probabilities exhibit a sharp increase in the late 2011. Since the increase in recession probabilities is closely preceded by the 2008-2009 recession, it provides empirical evidence of a potential double-dip recession in the period 2008-2011. It does not mean that the late 2011 will unequivocally be classified by the CEPR committee as an euro area recession. It means that the warning alarm system is pointing to a high risk of a new economic downturn that should be confirmed from the new releases of the economic indicators in the immediate future. Whether the euro-area economy will face a mild recession in the late 2011 , with relatively limited job losses as in the early 2000 s, or if it could become a very serious and deep recession as in 1992-1993 and 2008-2009 will depend on the severity of the incoming shocks hitting the system.

Let us focus on the informational content of the euro area economic indicators about the business cycle inferences. When the statistical agencies report a new release of an economic indicator, updating the outlook of the economy according to the new figure is not easy. The Markov-switching dynamic factor model facilitates this interpretation since it becomes a filtering rule which extracts the indicator's information about the state of the economy, by transforming the release into a probability of recession. Therefore, the output of the model is extremely easy to interpret.

\footnotetext{
${ }^{12}$ Notably, although the CEPR committee do not use a fixed rule to weight different data series used in the business cycle analysis, they give primary emphasis to GDP.
} 
To illustrate the usefulness of the Markov-switching model proposed in the paper to transform the information about the economic evolution contained in the business cycle indicators, we perform the following exercise. Suppose that we were in January 2006 and we had information about the economic indicators up to December 2005, which is clearly part of an expansion period. Now, we simulate potential outcomes of the following BNB release (from about -32 to 2 ) for January 2006. ${ }^{13}$ Then, we plot in Figure 3 (bottom line) the recession probabilities that would have been inferred from the data vintages that differ from each other only on the different generated BNB values. Following the same procedure, we repeat the exercise for January 2009, which is a within-recession month, and we also plot the simulated recession probabilities in Figure 3 (top line).

The nonlinear features that are accounted for by the model can be clearly detected by examining the inferred probabilities of recessions in January 2006 and January 2009. ${ }^{14}$ The curve associated to 2006 is clearly shifted down, which implies that the same BNB value contains very different information about the probability of an imminent recession depending on the period that we consider. In 2009, a BNB value of -20 would be associated with a probability of recession of almost 0.8 . However, the same value of BNB in 2006 would implied a much lower recession probability of about 0.3 . The intuition of this result is simple. To infer that the euro area business cycle deteriorates significantly, the negative evidence in the BNB must be worse in the curse of an expansion than it is required in recessions.

The Markov-switching dynamic factor specification implies richer relationships between the business cycle indicators and GDP previsions than those suggested by linear dynamic factor models. The new releases are converted into nonlinear inferences about the state of the business cycle which are then used to compute the forecasts of GDP growth by the model. To illustrate this nonlinear effect, we call the Kalman filter with the historical time series of all the data which are enlarged with each of the simulated values of BNB and obtain the GDP forecasts that are plotted in Figure 4. For extreme negative values

\footnotetext{
${ }^{13}$ Note that BNB is the earliest available indicator with current information about the first month of 2006.

${ }^{14} \mathrm{It}$ is worth recalling that we are using exclusively the data vintages that were available at the dates of the forecasts.
} 
of the indicator, the model would infer probabilities of recession that are close to one, which are then used to forecast GDP growth rates that are close to -1.5. As the values of BNB increase, the model predicts relatively better values of GDP growth which start to increase almost linearly with BNB. Around BNB values of -20 , for which Figure 3 showed a substantial decline in the inferred probability of recession, the expected responses of GDP to BNB changes increase dramatically. For values of BNB greater of about -9 , the inferred probabilities of recession become very low indicating that the economy would be in the expansionary phase. Since then, the expected GDP growth rates that correspond to larger BNB values become quasi linearly trended again.

\subsection{Real-time analysis}

\subsubsection{Forecasting euro area GDP growth}

The prediction of the aggregate economic activity in the euro area with dynamic factor models has enjoyed widespread popularity in the last decade. However, almost all of these studies evaluate the out-of-sample forecast errors from a model fitted to just a portion of the data set. Some of them perform pseudo real-time exercises which are also conducted with vintages that are extracted from final revised values of the data set, although preserving the pattern of the actual chronological order of the data releases. According to Stark and Croushore (2002), evaluating forecast errors by using latest-available data is questionable since measures of the forecast error can be deceptively lower when using latest-available data rather than real-time data. In accordance with this principle, we perform an actual real-time evaluation of the model which relies on data vintages that are constructed from the preliminary and partially revised data that were available at the time of the forecasts.

To perform a realistic assessment of the actual empirical reliability of the model proposed in this paper, we evaluate its real-time performance at forecasting euro area GDP and at tracking the euro area business cycles. The analysis is performed through a data set that consists of real-time vintages obtained from January 2nd, 2004 to November 24th, 2011, which ends up with 795 different vintages. Therefore, estimation, factor extraction, and GDP and recession probabilities forecasting were conducted in real-time each time 
that a new release for some of the thirteen indicators became available. ${ }^{15}$

Since we were interested in short-term forecasting, for each vintage we computed recursive blocks of 9-month GDP forecasts that include lagged, current and future GDP forecasts, which are usually known as backcasts, nowcasts and forecasts. As soon as the GDP figure for a given quarter becomes available, the nine-month forecasting horizon is moved forward conveniently. In addition, when some indicator is updated on a day of a given month $t$, we computed the recession probabilities as if an analyst had been using them to search for new turning points in month $t$, although the indicator may refer to an earlier month due to the typical lag with which some indicators become available.

The first forecasts were computed on January 2nd, 2004. To construct the backcast (2003.4), nowcast (2004.1) and forecast (2004.2), the data were standardized, and the parameters, factors and recession probabilities were estimated using only the data that were available on this day. Thus, the model is re-estimated and the 9-month GDP forecasts and the recession probabilities are computed using recursively increasing samples of data vintages until November 24th, 2011.

In the search of a benchmark model to compare the predictive accuracy of the Markovswitching dynamic factor model proposed in this paper, we consider that the Euro-STING model is an appropriate candidate. Remarkably, Camacho and Perez Quiros (2010) documented that this linear dynamic factor model is able to forecast the euro area GDP growth in real time not only better than standard ARIMA and random walks, but also as well as professional forecasters such as the European Commission's macroeconomic forecasts, the euro area GDP growth projection of DG ECFIN, the IFO-INSEE-INSAE economic forecast and the projections of the OECD Economic Outlook.

The accuracy of the linear and Markov-switching specifications in forecasting euro area growth is examined in Table 3. The entries show the Mean Squared Errors (MSE) which are the average of the deviations of the predictions from two alternative measures of GDP growth. The former is the real-time (labeled as real) GDP release, which is the first release of the second estimate of GDP published for a particular quarter. The latter is the final (labeled as final) releases of GDP that are available in the most updated dataset. To test

\footnotetext{
${ }^{15}$ The data vintages are kept fixed until the day that a new economic indicator is updated.
} 
whether the differences between the models are significant, we use the test proposed by Diebold and Mariano (1995), henceforth DM. Results for backcasts, nowcasts and forecasts appear horizontally. According to the table, in backcasting, forecasts from the nonlinear model outperform those from the linear model although the accuracy in nowcasting and forecasting reverses. Notably, according to the $p$-values of the DM tests the hypothesis of equal predictive accuracy cannot be rejected at conventional levels. This result implies that the nonlinear model is not a (statistically significant) worse predictor of the euro area GDP growth than the linear model.

\subsubsection{Forecasting euro area business cycle phases}

Despite the comparable accuracy in forecasting growth from the linear and nonlinear models, the main contribution of the latter is its ability to compute timely inferences about the euro-area business cycles. ${ }^{16}$ To examine the accuracy of the model to compute recession probabilities in real time, Figure 5 shows the filtered probabilities that would be inferred daily by a forecaster who used the information available at the day of the forecast from January, 1st 2008 to January, 15th 2010. Remarkably, this period includes the Great Recession.

Figure 5 helps to illustrate the usefulness of the model to compute early signals of upcoming recessions, which occurs whenever the filtered probabilities start to rise. According to this figure, in mid-July 2008 the probability of recession increased up to values that are very close to one. It is worth noting that this promptly signal of bad news about the state of the euro area economy represents a great improvement in the timing of turning points identifications with respect to other standard dating methods such as the two consecutive falls in GDP. In July, the latest available figure of second GDP was for 2008.1 (released July 9th) and it was still a positive and very high number (0.72) while the probability of recession reached a high record of 0.98. Since the GDP figures for the second and third quarters of 2008 were negative, if one considered that two consecutive falls of GDP growth

\footnotetext{
${ }^{16}$ Although we focused on forecasting growth, Perez-Quiros and Timmermann (2001) show that there are additional forecasting gains from Markov-switching specifications that rely on the forecasts of higher moments of the data, asymmetric risks and extreme values.
} 
mark the peak, the recession would not be formally identified before the publication day of the third quarter GDP, November 15th 2008. In addition, the CEPR committee did not confirm that the euro area entered a recession in the first quarter of 2008 until March, 31th 2009.

Figure 6 helps us to understand how the indicators help in the rapid turning point identification. The figure shows that both soft and hard indicators started the falls in early summer. The growth rates of IPI, INO and Exports were $-1.69,-4.69$ and -3.98 in May, IFO lost 3.8 points in June, and ESI and PMIM lost 5.1 and 1.8 points in July.

Figure 5 also helps to examine the timeliness in the identification of the green shoots that marked the 2009 trough. In particular, we define the green shoots as those values of the key economic indicators that produce low probabilities of being in recession at time $t$ after a period of recession. About mid-April 2009, the probability of recession dramatically dropped from values of about 0.8 to values close to zero. Accordingly, our model would have been able to find the popular green shoots in real time since it would have been able to establish the correct turning point date in real time. As in the case of the peak, we find evidence of a trough that marks the end of the recession before other standard dating methods since, in April 2009 the latest available figure of GDP growth was still very negative ( $-1.57 \%$ for the last quarter of 2008). Even later (15th of May 2009), the first quarter of 2009 was published with a more negative number $(-2.55 \%$ for the flash of 2009.1). The CEPR committee waited until October 4th, 2010 to announce that a trough in economic activity occurred in the second quarter of 2009.

Which are the mechanics behind these good signals that mark the changes in probabilities? When the probabilities of recession were still high at the beginning of April, the values of some soft indicators such as ESI and PMIM were 64.6 and 33.9, respectively. However, the following realizations since that date were 67.3 and 36.9 which implied significant improvements after several months of consecutive falls. In addition, the good news were confirmed by the hard indicators when they became available: IPI and INO increased from -0.46 and 0.10 in April to 1.64 and 1.42 in May, and Sales and Exports raised from -0.52 and -1.37 in May to 0.01 and 1.08 in June. Therefore, the Markov-switching dynamic factor model had unequivocally signaled in April 2009 that the through in the euro 
area had occurred. ${ }^{17}$ After a long winter, the green shoots of economic recovery in the euro area sprang up in May 2009 and the potential users of our model to monitor the business cycles would have had timely statistical evidence of this fact.

The deterioration in the debt crisis and the increasing fiscal tightening in many member states since summer 2011 led economic agents to believe that the aggregate performance of the euro area was quite sluggish and viewed this as the onset of a double-dip recession for the region as a whole, although with striking divergences across countries. Following the lines of Figure 5, we examine the ability of the model to detect the economic deterioration in Figure 7, which shows the filtered probabilities that would be inferred daily by a forecaster who used the information available at the day of the forecast from January, 15th 2011 to the latest available vintage on November, 24th 2011.

In the same way, we consider that the euro area enters a double-dip recession when there are high recession probabilities which are preceded by a short period of low recession probabilities observed after a recession. ${ }^{18}$ According to this figure, in mid-August 2011 the probability of recession increased up to values above 0.5. Again, this promptly signal of bad news represents a great improvement in the timing of turning points identifications. Figure 8 shows that although the hard indicators exhibited negative growths in several months of 2011 the sharp decrease took place in September, when the growth rates of IPI, INO, Sales and Exports became $-2.00 \%,-6.35 \%,-0.62 \%$ and $-1.01 \%$, respectively. The figure also shows the steadily declining in Purchasing Managers' Indices (PMI) and confidence indicators which exhibit a sharp decline in August 2011. On signs that weakness started appearing in the official data, the model increased the recession probabilities and has been foreshadowing a downturn since the summer. Notably, somewhat less negative news were coming from the soft indicators in the very late 2011. Waiting for hard indicators to confirm the potential tendency change, the recession probabilities diminished to 0.44 .

This result suggests some evidence of a double-dip recession in the last 2011. The warning alarm system provided by the model is pointing to a high risk of a new economic

\footnotetext{
${ }^{17}$ Interestingly, the number of searches of the term green shoots in Google trends shows a peak in May, a few days after the sharp reduction in the probability of recession.

${ }^{18}$ Note that, since expansions are long-lived in most industrialized countries, a double dip occurs when the recession probabilities become large soon after the preceding recession.
} 
downturn that should be confirmed from the new releases of the economic indicators that will become available in the immediate future. Therefore, although the method helps in performing early warning signals on the potential double-dip recession, the Dating Committee will wait to commit until some of the uncertainty about the severity of the incoming shocks hitting the system is resolved since it is costly to reverse their decisions if the euro-area recession is finally avoided.

According to these results, the Markov-switching dynamic factor model used in this paper could be very useful in monitoring the euro area economic evolution in real-time. Even for users that are not familiar with business cycle techniques, our nonlinear model facilitates the task of interpreting the new releases of the main euro area economic indicators by converting them into recession probabilities. One additional advantage of the model is that the process used to compute these inferences is transparent and easily replicated.

\section{Conclusion}

The Markov-switching dynamic factor model used in this paper to compute recession probabilities in real time can be viewed as formalizing the terms green shoots and double-dip recessions, which have became very popular since the Great Recession. The technique is successfully applied to euro area data to obtain a nonlinear indicator of the overall economic activity and business cycle inferences. The inferences show that estimates associated to high probabilities of negative values of the indicator are remarkably similar to the CEPR Committee's dating of the euro-area business cycle. The results show that the model could be used as an alternative objective algorithm for dating the euro area business cycles.

Recently, Hamilton (2011) acknowledged that it is hard to predict recessions in real time. To examine the ability of the model to compute euro-area business cycle inferences in real time, we collect for this article a real-time data set that has not been applied in any other analysis of real-time business cycle dating. The analysis developed in this paper show that the model identifies the CEPR turning points of the Great Recession in real time with remarkable accuracy, and with no instances of false positives. Therefore, we consider 
that using the daily updated probabilities which are computed from our computationally simple algorithm would facilitate the analysis of the day to day economic developments in the euro area. 


\section{Appendix A}

To illustrate how the matrices stated in the measurement and transition equations look like, let $0_{i, j}$ be a matrix of $(i \times j)$ zeroes, $I_{r}$ be the $r$-dimensional identity matrix, and $\otimes$ be the Kronecker product. According to the empirical application, let us assume that $m_{1}=0, m_{2}=m_{4}=6, m_{3}=2, r_{h}=4$, and $r_{s}=5$. For simplicity, let us assume that all variables are always observed at a monthly frequency.

In this example, the measurement equation, $Y_{t}=H_{t} h_{t}+w_{t}$, with $w_{t} \sim$ i.i.d.N $(0, R)$, can be expressed as

$$
\begin{aligned}
& Y_{t}=\left(\begin{array}{llllll}
y_{t}^{2 n d} & Z_{t}^{h^{\prime}} & Z_{t}^{s^{\prime}} & l_{t} & y_{t}^{1 s t} & y_{t}^{f}
\end{array}\right)^{\prime}, \\
& w_{t}=0_{r+4,1} \text {, } \\
& R_{t}=0_{r+4, r+4}, \\
& h_{t}=\left(f_{t}, \ldots, f_{t-11}, u_{1 t}, \ldots, u_{1 t-5}, v_{1 t}, v_{1 t-1}, \ldots, v_{r t}, v_{r t-1}, u_{2 t}, \ldots, u_{2 t-5}, e_{1 t}, e_{2 t}\right)^{\prime} .
\end{aligned}
$$

The matrix $H$ is in this case

$$
H=\left(\begin{array}{cccccccc}
H_{11} & 0_{1,6} & H_{12} & 0_{1,8} & 0_{1,10} & 0_{1,6} & 0 & 0 \\
H_{21} & 0_{r_{h}, 6} & 0_{r_{h}, 6} & H_{22} & 0_{r_{h}, 10} & 0_{r_{h}, 6} & 0_{r_{h}, 1} & 0_{r_{h}, 1} \\
H_{31} & H_{31} & 0_{r_{s}, 6} & 0_{r_{s}, 8} & H_{32} & 0_{r_{s}, 6} & 0_{r_{s}, 1} & 0_{r_{s}, 1} \\
H_{4} & 0_{1,6} & 0_{1,6} & 0_{1,8} & 0_{1,10} & H_{12} & 0 & 0 \\
H_{11} & 0_{1,6} & H_{12} & 0_{1,8} & 0_{1,10} & 0_{1,6} & 0 & 1 \\
H_{11} & 0_{1,6} & H_{12} & 0_{1,8} & 0_{1,10} & 0_{1,6} & 1 & 1
\end{array}\right),
$$

where

$$
\begin{aligned}
& H_{11}=\left(\begin{array}{llllll}
\frac{\beta_{1}}{3} & \frac{2 \beta_{1}}{3} & \beta_{1} & \frac{\beta_{1}}{3} & \frac{2 \beta_{1}}{3} & 0
\end{array}\right), \\
& H_{12}=\left(\begin{array}{llllll}
\frac{1}{3} & \frac{2}{3} & 1 & \frac{1}{3} & \frac{2}{3} & 0
\end{array}\right), \\
& H_{22}=I_{r_{h}} \otimes\left(\begin{array}{ll}
1 & 0
\end{array}\right), \\
& H_{32}=I_{r_{s}} \otimes\left(\begin{array}{ll}
1 & 0
\end{array}\right), \\
& H_{4}=\left(\begin{array}{llllll}
\frac{\beta_{4}}{3} & \frac{2 \beta_{4}}{3} & \beta_{4} & \frac{\beta_{4}}{3} & \frac{2 \beta_{4}}{3} & 0
\end{array}\right),
\end{aligned}
$$

$H_{21}$ is a $\left(r_{h} \times 6\right)$ matrix of zeroes whose first column is $\beta_{2}$, and $H_{31}$ is a $\left(r_{s} \times 6\right)$ matrix whose columns are $\beta_{3}$. 
Using the assumptions of the underlying example, the transition equation, $h_{t}=\Lambda_{s t}+$ $F h_{t-1}+\xi_{t}$, can be stated as follows. Let $Q$ be a diagonal matrix in which the entries inside the main diagonal are determined by the vector

$$
q=\left(\begin{array}{ccccccccccccc}
\sigma_{f}^{2} & 0_{1,11} & \sigma_{u_{1}}^{2} & 0_{1,5} & \sigma_{v_{1}}^{2} & 0 & \ldots & \sigma_{v_{r}}^{2} & 0 & \sigma_{u_{2}}^{2} & 0_{1,5} & \sigma_{e_{1}}^{2} & \sigma_{e_{2}}^{2}
\end{array}\right)^{\prime}
$$

The matrix $F$ becomes

$$
F=\left(\begin{array}{ccccccc}
a & 0_{12,6} & 0_{12,8} & 0_{12,10} & 0_{12,6} & 0 & 0 \\
0_{6,12} & b & 0_{6,8} & 0_{6,10} & 0_{6,6} & 0 & 0 \\
0_{8,12} & 0_{8,6} & c_{h} & 0_{8,10} & 0_{8,6} & 0 & 0 \\
0_{10,12} & 0_{10,6} & 0_{10,8} & c_{s} & 0_{10,6} & 0 & 0 \\
0_{6,12} & 0_{6,6} & 0_{6,8} & 0_{6,10} & d & 0 & 0 \\
0_{1,12} & 0_{1,6} & 0_{1,8} & 0_{1,10} & 0_{1,6} & 0 & 0 \\
0_{1,12} & 0_{1,6} & 0_{1,8} & 0_{1,10} & 0_{1,6} & 0 & 0
\end{array}\right),
$$

where

$$
\begin{aligned}
& a=\left(\begin{array}{cccccc}
0 & \ldots & 0 & \ldots & 0 & 0 \\
1 & \ldots & 0 & \ldots & 0 & 0 \\
\vdots & \vdots & \ddots & \vdots & \vdots & \vdots \\
0 & \ldots & 0 & \ldots & 1 & 0
\end{array}\right) \\
& b=\left(\begin{array}{cccc}
b_{1} & \ldots & b_{5} & b_{6} \\
1 & \ldots & 0 & 0 \\
\vdots & \ddots & \vdots & \vdots \\
0 & \ldots & 1 & 0
\end{array}\right), \\
& c_{h}=\left(\begin{array}{ccccc}
c_{11} & c_{12} & \ldots & 0 & 0 \\
1 & 0 & \ldots & 0 & 0 \\
\vdots & \vdots & \ddots & \vdots & \vdots \\
0 & 0 & \ldots & c_{r_{h} 1} & c_{r_{h} 2} \\
0 & 0 & \ldots & 1 & 0
\end{array}\right) \text {, }
\end{aligned}
$$




$$
\begin{gathered}
c_{s}=\left(\begin{array}{ccccc}
c_{1+r_{h} 1} & c_{1+r_{h} 2} & \ldots & 0 & 0 \\
1 & 0 & \ldots & 0 & 0 \\
\vdots & \vdots & \ddots & \vdots & \vdots \\
0 & 0 & \ldots & c_{r 1} & c_{r 2} \\
0 & 0 & \ldots & 1 & 0
\end{array}\right), \\
d=\left(\begin{array}{cccc}
d_{1} & \ldots & d_{5} & d_{6} \\
1 & \ldots & 0 & 0 \\
\vdots & \ddots & \vdots & \vdots \\
0 & \ldots & 1 & 0
\end{array}\right)
\end{gathered}
$$




\section{References}

[1] Alvarez, R., Camacho, M., and Perez Quiros, G. 2012. Finite sample performance of small versus large scale dynamic factor models. CEPR Working Paper 8867.

[2] Aruoba, B., Diebold, F., and Scotti, C. 2009a. Real-time measurement of business conditions. Journal of Business and Economic Statistics 7: 417-427.

[3] Aruoba, B., Diebold, F., and Scotti, C. 2009b. Updates on ADS Index Calculations. Mimeo. Available at www.philadelphiafed.org.

[4] Anas, J., Billio, M., Ferrara, L., and Lo Duca, M. 2006 A turning point chronology for the Euro-zone classical and growth cycle. In Growth and Cycle in the Euro-zone, eds. G.L. Mazzi and G. Savio, Palgrave-MacMillan, New-York.

[5] Boivin, J., and Ng, S. 2006. Are more data always better for factor analysis? Journal of Econometrics 132: 169-194.

[6] Camacho, M., and Perez Quiros, G. 2007. Jump-and-rest effect of U.S. business cycles. Studies in Nonlinear Dynamics and Econometrics 11(4): article 3.

[7] Camacho, M., and Perez-Quiros, G. 2010. Introducing the Euro-STING: Short Term INdicator of Euro Area Growth. Journal of Applied Econometrics, 25: 663-694.

[8] Camacho, M., Perez-Quiros, G., and Poncela, P. 2012a. Extracting nonlinear signals from several economic indicators. Bank of Spain Working Paper 1202.

[9] Camacho, M., Perez-Quiros, G., and Poncela, P. 2012b. Markov-switching dynamic factor models in real time. Bank of Spain Working Paper 1205.

[10] Chauvet, M. 1998. An economic characterization of business cycle dynamics with factor structure and regime switches. International Economic Review 39:969-996

[11] Chauvet, M., and Hamilton, J. 2006. Dating business cycle turning points in real time. In Nonlinear Time Series Analysis of Business Cycles, eds. C. Milas, P. Rothman, and D. Van Dijk. Amsterdam: Elsevier Science, pp. 1-54. 
[12] Chauvet, M., and Piger, J. 2008. A comparison of the real-time performance of business cycle dating methods. Journal of Business and Economic Statistics 26: 42-49.

[13] Coenen, C., Levin, A., and Wieland, V. 2005. Data uncertainty and the role of money as an information variable for monetary policy. European Economic Review 49: 9751006 .

[14] Diebold, F., and Mariano, R. 1995. Comparing predictive accuracy. Journal of Business and Economic Statistics 13: 253-263.

[15] European Commission. 2006. The joint harmonized EU programme of business and consumer surveys. Special Report No. 5/2006.

[16] Evans, M. 2005. Where are we now? Real time estimates of the macroeconomy. International Journal of Central Banking 1: 127-175.

[17] Hamilton, J. 2011. Calling recessions in real time. International Journal of Forecasting 27: 1006-1026.

[18] Kim, C. 1994. Dynamic Linear Models with Markov-Switching. Journal of Econometrics 60: 1-22.

[19] Kim, C., and Nelson, C. 1998. Business cycle turning points, a new coincident index, and tests of duration dependence based on a dynamic factor model with regime switching. Review of Economics and Statistics 80: 188-201.

[20] Kim, M., and Yoo, J. 1995. New index of coincident indicators: A multivariate Markov switching factor model approach. Journal of Monetary Economics 36: 607-630.

[21] Mariano, R., and Murasawa, Y. 2003. A new coincident index os business cycles based on monthly and quarterly series. Journal of Applied Econometrics 18: 427-443.

[22] Perez-Quiros, G., and Timmermann, A. 2001. Business cycle asymmetries in stock returns: Evidence from higher order moments and conditional densities. Journal of Econometrics 103: 259-306. 
[23] Stark T, Croushore D. 2002. Forecasting with a real-time data set for macroeconomists. Journal of Macroeconomics 24: 507-531.

[24] Stock, J., and Watson, M. 1991. A probability model of the coincident economic indicators. In Leading Economic Indicators: New Approaches and Forecasting Records, eds. K. Lahiri and G. Moore, Cambridge University Press.

[25] Stock, J., and Watson, M. 2011. Dynamic Factor Models. In Oxford Handbook of Forecasting, eds. M. Clements and D. Hendry, Oxford University Press. 